\title{
THE EIGENVALUES OF ONE-SPEED NEUTRONS IN A SLAB WITH FORWARD AND BACKWARD SCATTERING
}

\author{
Ökkeş EGE, Hakan ÖZTÜRK
}

\begin{abstract}
The eigenvalue spectrum is studied for one-speed neutrons in a slab with forward and backward scattering. First, the transport equation describing the interaction of neutrons in a system with general geometry is given. Then, the scattering function in transport equation is chosen as the forward-backward-isotropic (FBI) scattering model. The resultant transport equation is solved using the Legendre polynomials expansion ( $P_{N}$ method) and the Chebyshev polynomials of second kind expansion $\left(U_{N}\right.$ method) in neutron angular flux. Then, the $P_{\mathrm{N}}$ and $U_{\mathrm{N}}$ moments of the equations are obtained using the properties of the Legendre and the Chebyshev polynomials of the second kind. Finally, the eigenvalues for various values of the collision and scattering parameters are calculated using different orders of the presented methods and they are given in the tables for comparison.
\end{abstract}

Keywords: eigenvalue spectrum; forward and backward scattering; slab geometry; $U_{N}$ method; transport equation

\section{INTRODUCTION}

The criticality problem of a multiplying system is among the important problems in calculations of the neutron transport theory. There are many studies in literature about the solution of one-speed neutron transport equation with isotropic and anisotropic scattering for bare or reflected slabs and spheres. The scattering reactions from material atoms strongly affect the distribution and the interaction of the neutrons and thus the continuity of the fission chain reaction in the reactor system. Therefore, in the solution of the problems of neutron transport theory, the scattering of neutrons through the media should be as detailed as possible.

The series expansion of the angular flux or scattering function is commonly used in the problems of transport theory. In these approximations, neutron angular flux is expanded in two parts; the spatial and angular parts. The angular part of it is represented by an orthogonal polynomial. Among the orthogonal polynomials, the Legendre polynomials are the most widely used ones in the angular part of the neutron angular flux. This technique is known as the $P_{\mathrm{N}}$ method in literature. However, this does not indicate the validity of this method for all cases and problems. In some of the studies using $P_{\mathrm{N}}$ approximation, it is reported to be a rather poor representation of the angular flux especially near material boundaries [1]. Therefore, the Chebyshev polynomials of first kind ( $T_{\mathrm{N}}$ method) have been tried instead of Legendre polynomials. Ultimately in case of anisotropic scattering and extrapolated end point calculations, it was observed that better results were obtained in using the Chebyshev polynomials rather than the Legendre polynomials [2-4]. In recent years, since these polynomials are in the same family, i.e. Jacobi polynomials, the second kind of Chebyshev polynomials approximation ( $U_{\mathrm{N}}$ method) was used in criticality and diffusion length calculations for isotropic and anisotropic scattering in vacuum and reflecting boundary conditions. From those studies, it was observed that compatible results have been obtained in all cases [5-9]. As it is well known, an analytic or a numerical method can be used for all kinds of problems in science without hesitation. Therefore, in order to see the sustainability of the $U_{\mathrm{N}}$ method in different problems, it is still worth to use it in transport theory. In this study, eigenvalue spectrum for one-speed neutrons in a slab with forward, backward and isotropic scattering is studied using the present $U_{\mathrm{N}}$ method. The researchers in all areas may be interested in the solution algorithm followed in this study to apply it to their problems and thus an alternative method can be added to the literature.

The scattering function in transport equation is very important since it describes the interaction types of the neutron with the material boundaries. It should be as comprehensive as possible to represent the neutron interactions with the materials inside the system. There are many scattering functions in literature used for the neutron scattering function in transport equations. Among them, the forward-backward-isotropic (FBI) scattering model is one of the commonly used models in problems of transport theory $[10,11]$.

In the first part of the study, in order to compare the results obtained from the essential $U_{\mathrm{N}}$ method, the Legendre polynomials expansion $\left(P_{\mathrm{N}}\right.$ method) is used for the solution of the eigenvalue problem with forward and backward scattering. This is done because the results obtained from the $P_{\mathrm{N}}$ method are seen to be benchmark in many cases.

Then, in the second and the essential part of this study, the $U_{\mathrm{N}}$ method, in which the angular part of the neutron flux is represented by the second kind of Chebyshev polynomials, is used for the solution of the same problem.

In both methods, after expanding the angular part of the neutron flux in terms of the Legendre and the second kind of Chebyshev polynomials respectively, $P_{\mathrm{N}}$ and $U_{\mathrm{N}}$ moments of the equations are obtained using the orthogonality and the recurrence relations of those polynomials precisely. Then, eigenvalue equations are derived by offering a general solution for the neutron flux. The numerical results for the eigenvalues are calculated and thus the eigenvalue spectrum 
is obtained for using various values of the scattering parameters; collision and the forward and backward scattering parameters.

Calculated numerical results obtained by both methods are listed in the tables side by side. It can be observed from the derivations of the equations and the tabulated results that the $U_{\mathrm{N}}$ method is one of the most efficient methods among the polynomial expansion-based techniques with its rapid convergence and comparatively simple algebraic equations for the problems of transport theory.

\section{THE METHODS}

Many methods are developed for the solution of the neutron transport equation in literature. Some of them are stochastic and the others are deterministic. In this study, the approximation of the Chebyshev polynomials of second kind ( $U_{\mathrm{N}}$ method) is preferred since it is one of the most powerful methods among the polynomial expansion based techniques and it is hoped to be used in other problems of applied mathematics. In addition, the eigenvalue problem is derived using the spherical harmonics ( $P_{\mathrm{N}}$ method), i.e. a conventional, most commonly used and effective method for the solutions of the problems of transport theory. Therefore, the results obtained from both methods can easily be compared with each other.

\subsection{Solution with $P_{\mathrm{N}}$ Method}

The conservative form of the time-independent stationary transport equation for one-speed neutrons can be given as [10],

$$
\begin{aligned}
& \boldsymbol{\Omega} \cdot \nabla \psi(r, \boldsymbol{\Omega})+\sigma_{T} \psi(r, \boldsymbol{\Omega})= \\
& =c \sigma_{T} \int \psi\left(r, \boldsymbol{\Omega}^{\prime}\right) f\left(\boldsymbol{\Omega}^{\prime} \cdot \boldsymbol{\Omega}\right) \mathrm{d} \boldsymbol{\Omega}^{\prime}
\end{aligned}
$$

While $\boldsymbol{\Omega}^{\prime}$ represents the unit vector along the neutron velocity before (and $\boldsymbol{\Omega}$ after) a scattering collision, $\sigma_{T}$ represents the probability of all interactions of neutrons with materials; the total macroscopic cross-section. $\psi(r, \Omega)$ represents the neutron angular flux at position $r$ and direction $\Omega . c$ is the secondary neutron numbers and $f\left(\boldsymbol{\Omega} \cdot \boldsymbol{\Omega}^{\prime}\right)$ can be forwardbackward-isotropic (FBI) scattering model for the scattering function describing all kind of interaction of the neutrons with materials,

$$
\begin{aligned}
& f\left(\boldsymbol{\Omega}^{\prime} \cdot \boldsymbol{\Omega}\right)=\frac{1-\alpha-\beta}{4 \pi}+ \\
& +\frac{\alpha}{2 \pi} \delta\left(\boldsymbol{\Omega}^{\prime} \cdot \boldsymbol{\Omega}-1\right)+\frac{\beta}{2 \pi} \delta\left(\boldsymbol{\Omega}^{\prime} \cdot \boldsymbol{\Omega}+1\right)
\end{aligned}
$$

where $\alpha$ and $\beta$ coefficients can be seen as the forward and backward scattering probabilities, respectively; $0 \leq \alpha, \beta \leq 1$, $\alpha+\beta \leq 1[11]$.
First, the scattering function given in Eq. (2) is inserted in Eq. (1) and then the one-dimensional transport equation can be written as,

$$
\begin{aligned}
& \mu \frac{\partial \psi(x, \mu)}{\partial x}+\sigma_{T}(1-\alpha c) \psi(x, \mu)= \\
& =\frac{c \sigma_{T}}{2}(1-\alpha-\beta) \times \int_{-1}^{1} \psi\left(x, \mu^{\prime}\right) \mathrm{d} \mu^{\prime}+\beta c \sigma_{T} \psi(x,-\mu)
\end{aligned}
$$

The neutrons are assumed to have same speed and thus the energy and the slab is assumed to be finite, homogeneous and having a thickness of $2 a$ extending from $x=-a$ to $x=a$. The free space boundary and symmetry conditions are assumed:

$\psi(a, \mu)=0$

$\psi(x, \mu)=\psi(-x, \mu), \quad \mu>0$,

In $P_{\mathrm{N}}$ method, the neutron angular flux in onedimensional case is expanded in terms of the Legendre polynomials,

$\psi(x, \mu)=\sum_{m=0}^{\infty} \frac{2 m+1}{4 \pi} \Phi_{m}(x) P_{m}(\mu)$,
$-a \leq x \leq a,-1 \leq \mu \leq 1$.

The orthogonality and the recurrence relations of the Legendre polynomials are,

$$
\begin{aligned}
& \int_{-1}^{1} P_{n}(\mu) P_{m}(\mu) \mathrm{d} \mu=\frac{2}{2 n+1} \delta_{n, m}, \\
& (2 m+1) \mu P_{m}(\mu)=(m+1) P_{m+1}(\mu)+m P_{m}(\mu) .
\end{aligned}
$$

In the next solution process, Eq. (5) is inserted into Eq. (3). Then, the resultant equation is multiplied by $P_{n}(\mu)$ and integrated over $\mu \in[-1,1]$ by means of Eqs. (6) and (7). Ultimately, $P_{\mathrm{N}}$ moments of equations are obtained;

$$
\begin{aligned}
& (n+1) \frac{\mathrm{d} \Phi_{n+1}(x)}{\mathrm{d} x}+n \frac{\mathrm{d} \Phi_{n-1}(x)}{\mathrm{d} x}+ \\
& +\sigma_{T}(2 n+1)\left\{1-c\left[\alpha+(-1)^{n} \beta\right]\right\} \Phi_{n}(x)= \\
& =c \sigma_{T}(2 n+1)(1-\alpha-\beta) \Phi_{0}(x) \delta_{n, 0} .
\end{aligned}
$$

$n \geq 0$. Conventionally, the general solutions to Eq. (6) are employed of the form [10],

$$
\Phi_{n}(x)=G_{n}(v) \exp \left(\frac{\sigma_{T} x}{v}\right) .
$$


This solution is inserted into Eq. (6) for obtaining the eigenvalue equations and thus the eigenvalues,

$$
\begin{aligned}
& (n+1) G_{n+1}(v)+n G_{n-1}(v)+ \\
& +v(2 n+1)\left\{1-c\left[\alpha+(-1)^{n} \beta\right]\right\} G_{n}(v)= \\
& =v c(2 n+1)(1-\alpha-\beta) G_{0}(v) \delta_{n, 0},
\end{aligned}
$$

where $G_{-1}(v)=0$ and $G_{0}(v)=1$. As the requirement of the method one can set $G_{N+1}(v)=0$ to obtain the discrete and the continuum $v$ eigenvalues using various values of $c, \alpha$ and $\beta$. As an example for $P_{1}$ approximation, an expression for the eigenvalues can easily be derived from Eq. (10) by solving the coupled equations for $n=0$ and 1 together,

$$
v_{1,2}= \pm \frac{1}{\sqrt{3(1-c)[1-c(\alpha-\beta)]}} .
$$

\subsection{Solution with $U_{\mathrm{N}}$ Method}

In some of recent studies, the following function is used as angular flux in transport equation and compatible results are reported to be obtained in various problems of neutron transport $[5,6]$ :

$$
\begin{aligned}
& \psi(x, \mu)=\frac{2}{\pi} \sqrt{1-\mu^{2}} \sum_{n=0}^{N} \Phi_{n}(x) U_{n}(\mu), \\
& -a \leq x \leq a, \quad-1 \leq \mu \leq 1
\end{aligned}
$$

As in the derivation of the equations in $P_{\mathrm{N}}$ method, prior to starting the applications, the orthogonality and recurrence relations of the Chebyshev polynomials of second kind are needed [12],

$$
\begin{aligned}
& \int_{-1}^{1} U_{n}(\mu) U_{m}(\mu) \sqrt{1-\mu^{2}} \mathrm{~d} \mu=\frac{\pi}{2} \delta_{n, m}, \\
& U_{n+1}(\mu)-2 \mu U_{n}(\mu)+U_{n-1}(\mu)=0 .
\end{aligned}
$$

Indeed, the same procedure with the $P_{\mathrm{N}}$ method is valid for the $U_{\mathrm{N}}$ method. In order to obtain the $U_{\mathrm{N}}$ moments of the angular flux Eq. (12) is needed to be inserted into Eq. (3). Then if one multiplies the resultant equation by $U_{m}(\mu)$ and integrates it over $\mu \in[-1,1]$ :

$$
\begin{aligned}
& \frac{\mathrm{d} \Phi_{1}(x)}{\mathrm{d} x}+2 \sigma_{T}(1-c) \Phi_{0}(x)=0 \\
& \frac{\mathrm{d} \Phi_{n+1}(x)}{\mathrm{d} x}+\frac{\mathrm{d} \Phi_{n-1}(x)}{\mathrm{d} x}+2 \sigma_{T}\left\{1-c\left[\alpha+(-1)^{n} \beta\right]\right\} \Phi_{n}(x)= \\
& =c \sigma_{T}(1-\alpha-\beta)\left\{\frac{1+(-1)^{n}}{n+1} \Phi_{0}(x)\right\},
\end{aligned}
$$

$n \geq 1$. The ansatz solution for neutron flux given in Eq. (9) is substituted into Eqs. (15). One can easily obtain the following system of algebraic equations for $G_{n}(v)$ :

$$
\begin{aligned}
& G_{1}(v)=-2 v(1-c) G_{0}(v), \\
& G_{n+1}(v)+G_{n-1}(v)+2 v\left\{1-c\left[\alpha+(-1)^{n} \beta\right]\right\} G_{n}(v)= \\
& =v c(1-\alpha-\beta)\left\{\frac{1+(-1)^{n}}{n+1} G_{0}(v)\right\}, \quad n \geq 1,
\end{aligned}
$$

By following the same procedure described in $P_{\mathrm{N}}$ approximation, the discrete and continuum $v$ eigenvalues are computed by setting $G_{N+1}(v)=0$ for various values of $c, \alpha$ and $\beta$. As an example for $U_{1}$ approximation, an expression for the eigenvalues can easily be derived from Eqs. (16),

$$
v_{1,2}= \pm \frac{1}{2 \sqrt{(1-c)[1-c(\alpha-\beta)]}}
$$

After computing the discrete eigenvalues, since all eigenvalues correspond to a linear independent eigenfunction, the linear combination of the eigenfunctions can be written as the $n^{\text {th }}$ Chebyshev moment of angular flux for odd numbers of $N$,

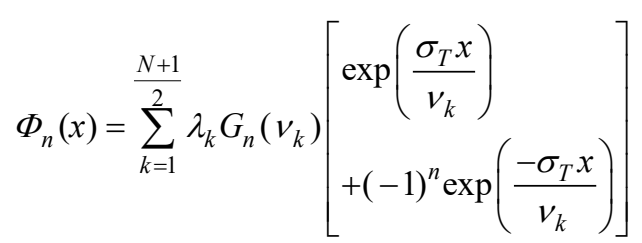

$n=1, \ldots, N,-a \leq x \leq a$

where $\lambda_{k}$ are the linear combination coefficients and they can be determined from the physical boundary conditions. The parity property of $G_{n}(-v)=(-1)^{n} G_{n}(v)$ is also used.

\section{NUMERICAL RESULTS}

The $U_{\mathrm{N}}$ method which is one of the most powerful methods improved for the solution procedure of the transport equation is used for the solution of the eigenvalue problem for one-speed neutrons in a homogeneous slab with forward and backward scattering. Before starting to apply the $U_{\mathrm{N}}$ method to the problem, in order to establish a benchmark list for the comparison of the results, first the conventional $P_{\mathrm{N}}$ method is applied to the transport equation for the solution of the problem. The function given in Eq. (5) is used for the neutron angular flux in Eq. (3) in $P_{\mathrm{N}}$ method. At the end of the operation of the conventional $P_{\mathrm{N}}$ method to the transport equation, Eq. (10) is obtained for calculating the eigenvalues for various values of the scattering parameters. This operation has been done to illustrate the similarity of the methodology derived by both methods. This similarity is expected since the functions used in both methods have the same definition interval and they are in 
the same polynomial family.

Then, in the main part of this study, the function involving the Chebyshev polynomials of second kind given in Eq. (12) is used for the neutron angular flux in transport equation together with the FBI scattering model given in Eq. (2) to obtain the $U_{\mathrm{N}}$ moments of equations. Thereafter, a general solution is employed to the moment equations and analytic expressions for a system of algebraic equations of the eigenvalues are obtained and they are given in Eqs. (16). Numerical results for the eigenvalues are calculated by setting $G_{N+1}(v)=0$ in Eqs. (16) as in $P_{\mathrm{N}}$ approximation for various values of the collision parameter $c$, forward scattering parameter $\alpha$ and backward scattering parameter $\beta$. Although these results are computed up to an order of $N=5$, the order of approximation can be extended to $N>5$. In all cases, the total macroscopic cross section is assumed to be its normalized value, $\sigma_{T}=1 \mathrm{~cm}^{-1}$.

\section{CONCLUSIONS}

The numerical results for the eigenvalues are computed for the values of $c$ ranging from 0.90 to 1.20 . They are calculated in the cases of isotropic scattering $(\alpha=0.0 ; \beta=$ $0.0)$, forward scattering $(\alpha=0.3 ; \beta=0.0)$ and backward scattering $(\alpha=0.0 ; \beta=0.3)$ using both $P_{\mathrm{N}}$ and $U_{\mathrm{N}}$ methods.

Table 1 Eigenvalue spectrum for isotropic scattering $(\alpha=0.0 ; \beta=0.0)$
\begin{tabular}{|c|c|c|c|c|c|c|}
\hline$c$ & $P_{1}$ & $U_{1}$ & $P_{3}$ & $U_{3}$ & $P_{5}$ & $U_{5}$ \\
\hline 0.90 & 1.82574 & 1.58114 & 0.48657 & 0.41811 & 0.30333 & 0.27959 \\
& & & 1.90273 & 1.89082 & 0.80588 & 0.74313 \\
& & & & & 1.90320 & 1.90252 \\
\hline 0.95 & 2.58199 & 2.23607 & 0.49688 & 0.42556 & 0.30775 & 0.28348 \\
& & & 2.63507 & 2.62721 & 0.81133 & 0.74840 \\
& & & & & 2.63515 & 2.63493 \\
\hline 0.99 & 5.77350 & 5.00000 & 0.50506 & 0.43152 & 0.31132 & 0.28664 \\
& & & 5.79673 & 5.79343 & 0.81526 & 0.75231 \\
& & & & & 5.79673 & 5.79671 \\
\hline 1.01 & $5.77350 \mathrm{i}$ & $5.00000 \mathrm{i}$ & $5.75054 \mathrm{i}$ & $5.75373 \mathrm{i}$ & $5.75054 \mathrm{i}$ & $5.75052 \mathrm{i}$ \\
& & & 0.50912 & 0.43450 & 0.31311 & 0.28823 \\
& & & & & 0.81709 & 0.75417 \\
\hline 1.10 & $1.82574 \mathrm{i}$ & $1.58114 \mathrm{i}$ & $1.75703 \mathrm{i}$ & $1.76555 \mathrm{i}$ & $1.75665 \mathrm{i}$ & $1.75620 \mathrm{i}$ \\
& & & 0.52692 & 0.44777 & 0.32127 & 0.29547 \\
& & & & & 0.82437 & 0.76176 \\
\hline 1.20 & $1.29099 \mathrm{i}$ & $1.11803 \mathrm{i}$ & $1.20016 \mathrm{i}$ & $1.20976 \mathrm{i}$ & $1.19830 \mathrm{i}$ & $1.19723 \mathrm{i}$ \\
& & & 0.54547 & 0.46209 & 0.33041 & 0.30364 \\
& & & & & 0.83089 & 0.76887 \\
\hline
\end{tabular}

Table 2 Eigenvalue spectrum for forward scattering $(\alpha=0.3 ; \beta=0.0)$
\begin{tabular}{|c|c|c|c|c|c|c|}
\hline$c$ & $P_{1}$ & $U_{1}$ & $P_{3}$ & $U_{3}$ & $P_{5}$ & $U_{5}$ \\
\hline 0.90 & 2.13687 & 1.85058 & 0.65609 & 0.56524 & 0.41111 & 0.37910 \\
& & & 2.26245 & 2.24245 & 1.09787 & 1.01227 \\
& & & & & 2.26390 & 2.26229 \\
\hline 0.95 & 3.05352 & 2.64443 & 0.68920 & 0.59103 & 0.42794 & 0.39429 \\
& & & 3.14223 & 3.12885 & 1.13179 & 1.04385 \\
& & & & & 3.14250 & 3.14197 \\
\hline 0.99 & 6.88592 & 5.96338 & 0.71721 & 0.61294 & 0.44230 & 0.40726 \\
& & & 6.92541 & 6.91977 & 1.15912 & 1.06958 \\
& & & & & 6.92542 & 6.92537 \\
\hline 1.01 & $6.91549 \mathrm{i}$ & $5.98899 \mathrm{i}$ & $6.87613 \mathrm{i}$ & $6.88158 \mathrm{i}$ & $6.87613 \mathrm{i}$ & $6.87608 \mathrm{i}$ \\
& & & 0.73170 & 0.62431 & 0.44978 & 0.41402 \\
& & & & & 1.17285 & 1.08259 \\
\hline 1.10 & $2.23050 \mathrm{i}$ & $1.93167 \mathrm{i}$ & $2.10923 \mathrm{i}$ & $2.12318 \mathrm{i}$ & $2.10778 \mathrm{i}$ & $2.10666 \mathrm{i}$ \\
& & & 0.80037 & 0.67895 & 0.48622 & 0.44699 \\
& & & & & 1.23547 & 1.14242 \\
\hline 1.20 & $1.61374 \mathrm{i}$ & $1.39754 \mathrm{i}$ & $1.45011 \mathrm{i}$ & $1.46395 \mathrm{i}$ & $1.44269 \mathrm{i}$ & $1.43994 \mathrm{i}$ \\
& & & 0.88174 & 0.74581 & 0.53228 & 0.48889 \\
& & & & & 1.30738 & 1.21169 \\
\hline
\end{tabular}

In Tab. 1, the eigenvalues for isotropic scattering are given. While the eigenvalues for forward scattering are given in Tab. 2 , the eigenvalues for backward scattering are given in Tab. 3 . As can be followed from all tables, all eigenvalues are real when $c$ $<1$. These eigenvalues can be used for diffusion theory (diffusion coefficient or diffusion length) or calculation of scalar flux. On the other hand, imaginary eigenvalues are seen when $c>1$ as expected. Since this situation corresponds to supercritical reactor, the eigenvalues for $c>1$ are used in criticality problems. It is seen from tables 1 through 3 , the numerical results for the eigenvalues obtained from both methods exhibit the same behaviour. Although it is not shown in the results in tables, one can easily understand that the tabulated eigenvalues are in pairs as they are felt in Eq. (11) and Eq. (17).

Table 3 Eigenvalue spectrum for backward scattering $(\alpha=0.0 ; \beta=0.3)$

\begin{tabular}{|c|c|c|l|l|l|l|}
\hline$c$ & $P_{1}$ & $U_{1}$ & $P_{3}$ & $U_{3}$ & $P_{5}$ & \multicolumn{1}{c|}{$U_{5}$} \\
\hline 0.90 & 1.62008 & 1.40303 & 0.49742 & 0.42854 & 0.31169 & 0.28742 \\
& & & 1.71530 & 1.70013 & 0.83236 & 0.76746 \\
& & & & & 1.71640 & 1.71517 \\
\hline 0.95 & 2.27773 & 1.97257 & 0.51410 & 0.44087 & 0.31922 & 0.29412 \\
& & & 2.34390 & 2.33392 & 0.84424 & 0.77864 \\
& & & & & 2.34410 & 2.34371 \\
\hline 0.99 & 5.06955 & 4.39036 & 0.52803 & 0.45126 & 0.32563 & 0.29983 \\
& & & 5.09863 & 5.09448 & 0.85337 & 0.78744 \\
& & & & & 5.09863 & 5.09860 \\
\hline 1.01 & $5.05786 \mathrm{i}$ & $4.38024 \mathrm{i}$ & $5.02908 \mathrm{i}$ & $5.03306 \mathrm{i}$ & $5.02907 \mathrm{i}$ & $5.02904 \mathrm{i}$ \\
& & & 0.53515 & 0.45661 & 0.32897 & 0.30281 \\
& & & & & 0.85780 & 0.79179 \\
\hline 1.10 & $1.58312 \mathrm{i}$ & $1.37102 \mathrm{i}$ & $1.49705 \mathrm{i}$ & $1.50695 \mathrm{i}$ & $1.49602 \mathrm{i}$ & $1.49523 \mathrm{i}$ \\
& & & 0.56807 & 0.48189 & 0.34510 & 0.31726 \\
& & & & & 0.87689 & 0.81085 \\
\hline 1.20 & $1.10702 \mathrm{i}$ & $0.95871 \mathrm{i}$ & $0.99476 \mathrm{i}$ & $1.00426 \mathrm{i}$ & $0.98968 \mathrm{i}$ & $0.98779 \mathrm{i}$ \\
& & & 0.60487 & 0.51162 & 0.36514 & 0.33537 \\
& & & & & 0.89686 & 0.83121 \\
\hline
\end{tabular}

In this study, the neutrons are assumed to be scattered isotropically in forward and backward directions in a homogeneous slab. Eq. (12) which is expanded in a series of the Chebyshev polynomials of second kind is used as the angular neutron flux in transport equation. The derived equations for the $U_{\mathrm{N}}$ moments are solved for the eigenvalue spectrum of the neutrons. These eigenvalues can be extended for requested collision and scattering parameters and they can be used in many problems of transport theory such as the criticality calculations, diffusion theory or scalar flux calculations. The method used in this study has already been used in other studies and the applicability and validity of it were proven from the results [5-7]. In addition, the traditional $P_{\mathrm{N}}$ method is also applied to the problem and the results obtained from this method are given in tables for comparison. From the tabulated results, one can easily realize that the $U_{\mathrm{N}}$ method is an effective method for the transport equation and thus it can be used in other problems of science and engineering. In near future, this study can be extended to the cases with anisotropic scattering and reflecting boundary conditions.

\section{REFERENCES}

[1] Lee, C. E. \& Dias, M. P. (1984). Analytical solutions to the moment transport equations-I; one-group one-region slab and sphere criticality. Ann. Nucl. Energy, 11, 515-530. https://doi.org/10.1016/0306-4549(84)90076-8 
[2] Aspelund, O. (1958). On a new method for solving the (Boltzmann) equation in neutron transport theory. PICG, 16, 530-534.

[3] Conkie, W. R. (1959). Polynomial approximations in neutron transport theory. Nucl. Sci. Eng., 6, 260-266. https://doi.org/10.13182/NSE59-A28841

[4] Yabushita, S. (1961). Tschebyscheff polynomials approximation method of the neutron transport equation. $J$. Math. Phys., 2, 543-549. https://doi.org/10.1063/1.1703739

[5] Öztürk, H., Anl, F., \& Güngör, S. (2007). Application of the $\mathrm{U}_{\mathrm{N}}$ method to the reflected critical slab problem for one-speed neutrons with forward and backward scattering. Kerntechnik, 72, 74-76. https://doi.org/10.3139/124.100321

[6] Öztürk, H. (2008). The reflected critical slab problem for onespeed neutrons with strongly anisotropic scattering. Kerntechnik, 73, 66-74. https://doi.org/10.3139/124.100532

[7] Öztürk, H. (2008). Study of the effect of anisotropic scattering on the critical slab problem in neutron transport theory using Chebyshev polynomials. Kerntechnik, 73, 284-287. https://doi.org/10.3139/124.100572

[8] Öztürk, H. (2011). Modified $U_{\mathrm{N}}$ method for the reflected critical slab problem with forward and backward scattering. Kerntechnik, 76, 142-145. https://doi.org/10.3139/124.110126

[9] Öztürk, H. (2014). The effect of strongly anisotropic scattering on the critical size of a slab in one-speed neutron transport theory: Modified $U_{\mathrm{N}}$ method. Ann. Nucl. Energy, 65, 24-29. https://doi.org/10.1016/j.anucene.2013.10.021

[10] Davison, B. (1958). Neutron transport theory. London: Oxford University Press. https://doi.org/10.1063/1.3062414

[11] Sahni, D. C., Sjöstrand, N. G., \& Garis, N. S. (1992). Criticality and time eigenvalues for one-speed neutrons in a slab with forward and backward scattering. J. Phys. D: Appl. Phys., 25, 1381-1389. https://doi.org/10.1088/0022-3727/25/10/001

[12] Arfken, G. (1985). Mathematical methods for physicists. London: Academic Press, Inc.

\section{Authors' contacts:}

Ökkeş EGE, MsC student

Osmaniye Korkut Ata University,

Faculty of Arts and Sciences,

Department of Physics,

Osmaniye, 80000, Turkey

Tel. +903288251818

E-mail: okkesege@gmail.com

Hakan ÖZTÜRK, PhD, Full Professor

(Corresponding author)

Osmaniye Korkut Ata University,

Faculty of Arts and Sciences,

Department of Physics,

Osmaniye, 80000, Turkey

Tel. +90 3288251818

E-mail: hakanozturk@osmaniye.edu.tr 